\title{
Microagulhamento em cicatrizes de queimadura: revisão de literatura
}

\author{
Microneedling in burn scars: literature review \\ Microneedling en cicatrices de quemaduras: revisión de la literatura
}

\author{
Rayssa Silva do Espirito Santo \\ ORCID: https://orcid.org/0000-0002-7804-3823 \\ Centro Universitário da Amazônia, Brasil \\ E-mail: rs6486070@gmail.com \\ Jeciane de Melo Roberto \\ ORCID: https://orcid.org/0000-0002-8835-0456 \\ Centro Universitário da Amazônia, Brasil \\ E-mail: jecianemello19@gmail.com \\ Naira Patrícia Castro de Oliveira \\ ORCID: https://orcid.org/0000-0003-1896-7693 \\ Centro Universitário da Amazônia, Brasil \\ E-mail: nairacastro@oultlook.com
}

\begin{abstract}
Resumo
Aproximadamente 1 milhão de pessoas sofrem queimaduras todos os anos, elas causam danos parciais ou até mesmo totais à pele. As cicatrizes causadas pelo tipo de lesão mencionada fazem com que os pacientes recorram a tratamentos que melhorem o aspecto das cicatrizes. O microagulhamento, também conhecido como indução percutânea de colágeno preserva a epiderme e reorganizaas fibras colágenas normalizando o aspecto do tecido lesionado. Portanto, o trabalho tem como objetivo, identificar através de uma revisão literária, os benefícios e resultados do uso da técnica de microagulhamento em cicatrizes de vítimas de queimaduras, por meio da fisioterapia dermatofuncional. Desse modo, este estudo trata-se de uma revisão de literatura, por meio de um levantamento em que buscou-se estudos que comprovem a eficácia da técnica do microagulhamento. De acordo com os resultados apresentados neste estudo, foram observados resultados positivos do microagulhamento em cicatrizes de queimaduras. Sendo assim, apesar de o microagulhamento apresentar-se como uma técnica nova no mercado, nota-se que vem ganhando o seu espaço e provando ser promissora e eficaz no tratamento das cicatrizes de queimaduras, promovendo a melhora do aspecto estético, assim como também da autoestima dos pacientes.
\end{abstract}

Palavras-chave: Microagulhamento; Cicatriz; Queimadura; Fisioterapia dermatofuncional.

\begin{abstract}
Approximately 1 million people suffer burns every year, they cause partial or even total damage to the skin. Scars caused by the type of injury mentioned make patients resort to treatments that improve the appearance of the scars. Microneedling, also known as percutaneous collagen induction, preserves the epidermis and reorganizes collagen fibers, normalizing the appearance of the injured tissue. Therefore, the work aims to identify, through a literature review, the benefits and results of the use of the microneedling technique in scars of burn victims, through dermatofunctional physiotherapy. Thus, this study is a literature review, through a survey in which studies were sought to prove the effectiveness of the microneedling technique. According to the results presented in this study, positive results of microneedling were observed in burn scars. Therefore, although microneedling presents itself as a new technique in the market, it is noted that it has been gaining space and proving to be promising and effective in the treatment of burn scars, promoting the improvement of the aesthetic aspect, as well as the patients' self-esteem.
\end{abstract}

Keywords: Microneedling; Scar; Burn; Dermatofunctional physiotherapy.

\section{Resumen}

Aproximadamente 1 millón de personas sufren quemaduras cada año, que provocan daños parciales o incluso totales en la piel. Las cicatrices provocadas por el tipo de lesión mencionado hacen que los pacientes recurran a tratamientos que mejoren el aspecto de las cicatrices. La microaguja, también conocida como inducción de colágeno percutáneo, preserva la epidermis y reorganiza las fibras de colágeno, normalizando la apariencia del tejido lesionado. Por tanto, el trabajo tiene como objetivo identificar, a través de una revisión de la literatura, los beneficios y resultados del uso de la técnica de microagujas en cicatrices de víctimas de quemaduras, a través de fisioterapia dermatofuncional. Así, este estudio es una revisión de la literatura, a través de una encuesta en la que se buscaron estudios para demostrar la efectividad de la técnica de microagujas. Según los resultados presentados en este estudio, se observaron resultados positivos de microagujas en cicatrices de quemaduras. Por tanto, aunque la microaguja se presenta como una nueva técnica en el 
mercado, se nota que ha ido ganando espacio y resultando prometedora y eficaz en el tratamiento de las cicatrices de quemaduras, favoreciendo la mejora del aspecto estético, así como de los pacientes. ' autoestima.

Palabras clave: Microneedling; Cicatriz; Quemar; Fisioterapia dermatofuncional.

\section{Introdução}

A pele é o órgão mais afetado pela queimadura. Ela tem como função a sensação, proteção, termorregulação e secreção. Os receptores sensitivos estão localizados nela, reconhecidos por meio de quatro submodalidades somestésicas, sendo elas a: dor, tato, temperatura e pressão. Conforme o receptor é estimulado, impulsos nervosos são gerados e enviados para o córtex cerebral, o qual é interpretado. A estimulação e combinação de estímulos resultam em sensações como queimação, prurido e cócegas, identificadas pelo cérebro (Dangello \& Fattini, 2002).

O sistema tegumentar é constituído pela pele e tela subcutânea, juntamente com os anexos cutâneos. O tegumento recobre toda a superfície do corpo e é constituído por uma porção epitelial, a epiderme, e uma porção conjuntiva, a derme (Guirro \& Guirro 2004).

Sendo assim, a queimadura é um problema real, no qual por ano aproximadamente um milhão de pessoas são atingidas, que podem resultar em sequelas, como por exemplo, as cicatrizes e, em casos mais graves, levar os indivíduos até mesmo a morte. No Brasil, estima-se que, por ano, pelo menos 1.000.000 indivíduos sofram queimaduras sem distinção de sexo, idade, procedência ou classe social, provocando um forte impacto econômico, considerando-se o tempo despendido para o tratamento, respondendo por cerca de 100.000 atendimentos hospitalares e até 2.500 óbitos anuais (Lamberti, et al., 2014).

A queimadura caracteriza-se pela ação direta ou indireta do calor sobre o corpo humano devido o contato com chamas, líquido aquecidos, frio extremo, substancias químicas e radiação, sendo classificadas de acordo com a profundidade em queimaduras de primeiro, segundo e terceiro grau (Serra, et al., 2006).

A queimadura de espessura superficial ou de $1^{\circ}$ grau é a que atinge somente a camada epidérmica, a qual é clinicamente caracterizada por eritema, edema e dor. Na evolução há prurido e descamação. A queimadura de espessura parcial superficial ou de $2^{\circ}$ grau superficial, é a que atinge toda a epiderme e parte da derme, em especial a derme papilar, conservando parcialmente os apêndices epidérmicos. Clinicamente, é caracterizada por vesícula, eritema, dor acentuada, edema e apresenta a base das bolhas avermelhada. A pele sofre processo de restauração completa dentro de 7 a 10 dias. Já a queimadura de espessura parcial profunda ou de $2^{\circ}$ grau profundo é a que atinge toda a epiderme e a derme mais profundamente, até a derme reticular, mantendo ainda parcialmente os apêndices epidérmicos. Contudo, clinicamente, não apresenta dor e a base das bolhas é esbranquiçada. Pode ocorrer restauração, mas há alterações de textura e pigmentação (Ferreira, 2007).

As lesões pós-queimadura são classificadas em: (a) sequelas funcionais, aquelas que limitam qualquer função de um segmento após uma queimadura, e (b) as sequelas estéticas ou não funcionais, que são as que não comprometem a funcionalidade, mas que podem interferir nos aspectos sociais decorrentes da nova aparência (Vana, 2013).

Dessa forma a intervenção da fisioterapia dermatofuncional nesse tipo de lesão é de muita importância, visando a diminuição de sequelas ocasionadas pela lesão, na melhoria da qualidade de vida e da integração na sociedade.

O fisioterapeuta possui aptidões específicas, certas metodologias e linhas de conhecimentos que irão atuar no tratamento. Para cada fase cronológica do avanço da queimadura, o fisioterapeuta possui um recurso específico que irá permitir que a lesão cicatrize de maneira correta, evitando complicações futuras (Guirro \& Guirro, 2007).

Como forma de tratamento o microagulhamento é uma técnica utilizada para várias disfunções estéticas na pele. A técnica é realizada por meio da perfuração do estrato córneo, sem ocasionar danos à epiderme, liberando fatores de crescimento que irão incentivar a produção de colágeno e elastina da derme papilar (Souza, 2015).

O Colágeno é o componente fibrilar principal do tecido conectivo e a proteína extracelular mais importante do corpo humano (Harris, 2009). 
A indução percutânea de colágeno (IPC) ou microagulhamento, inicia-se com a perda da integridade da barreira cutânea, tendo como alvo a dissociação dos queratinócitos, liberando as citocinas, como a interleucina-1 $\alpha$, predominantemente, além da interleucina-8, interleucina-6, TNF- $\alpha$ e GM-CSF, resultando em vasodilatação dérmica e migração de queratinócitos para restaurar o dano epidérmico. Existem três fases do processo de cicatrização, seguindo o trauma com as agulhas, que podem ser bem delineadas, didaticamente: na primeira, a de injúria, ocorre liberação de plaquetas e neutrófilos responsáveis pela liberação de fatores de crescimento com ação sobre os queratinócitos e os fibroblastos como os fatores de crescimento de transformação $\alpha$ e $\beta$ (TGF- $\alpha$ e TGF- $\beta$ ), o fator de crescimento derivado das plaquetas (PDGF), a proteína III ativadora do tecido conjuntivo e o fator de crescimento do tecido conjuntivo (Lima, et al., 2013).

Os rolos são de utilização única conforme determinados pelos seus fabricantes e pela própria Agência Nacional de Vigilância Sanitária (Anvisa) mediante a resolução n 2.605 de 11 de agosto de 2006, uma vez que se enquadram como agulhas com componentes plásticos não desmontáveis, portanto, proibidos de serem reprocessados e devem ser descartados em recipientes específicos para materiais perfurocortantes (Brasil, 2006 \& Negrão, 2015).

O procedimento com agulhas de até $1 \mathrm{~mm}$ consegue ser efetuado sem bloqueio anestésico ou com anestesia tópica, porém, acima desse tamanho já é necessário um bloqueio complementado por anestesia infiltrativa ou anestesia tópica mais forte (Lima, et al., 2013 \& Lima, 2015 \& Negrão, 2015). A utilização do creme anestésico Emla pode ajudar no alívio da dor e na realização do procedimento (Doddaballapur, 2009).

A pressão vertical exercida sobre o roller, não deve ultrapassar $6 \mathrm{~N}$, pois uma força superior pode levar danos as estruturas anatômicas mais profundas e mais dor que o esperado ao paciente. Recomenda-se posicionar o aparelho entre os dedos indicador e polegar como se estivesse segurando um "háshi" e controlar a força exercida com o polegar. Os movimentos de vai e vem devem guiar-se por padrão uniforme de petéquias em toda a área tratada. Para isso, entre dez (10) a quinze (15) passadas em uma mesma direção e pelo menos 4 cruzamentos na área de rolagem são suficientes. Teoricamente quinze (15) passadas provocam dano de 250-300 punturas $/ \mathrm{cm}^{3}$ (Moren, 2009).

O microagulhamento, de uma forma geral, é bem tolerado pelos clientes, porém um eritema pode ser observado após o procedimento, desaparecendo entre dois a três dias (Doddaballapur, 2009 \& Fabbrocini, et al., 2009 \& Liebl \& Kloth, 2012 \& Kalil, et al., 2015). O eritema na pele caucasiana diminui em 50\% após 4 a 6 horas do procedimento realizado. Máscaras geladas com ácido hialurônico podem ser utilizadas para reduzir o edema em 50\% em 30 minutos. Um edema visível após o microagulhamento é atípico, porém, um pequeno inchaço generalizado pode ser observado, mas o mesmo some em 48 horas (Liebl \& Kloth, 2012). O cliente pode retornar as suas atividades no dia seguinte (Doddaballapur, 2009 \& Kalil, et al., 2015b). O tempo de intervalo entre as sessões são em média de quatro semanas, pois, as novas fibras de colágeno levam um tempo para maturarem (Doddaballapur, 2009 \& Negrão, 2015).

\section{Metodologia}

O presente estudo trata-se de uma revisão de literatura de caráter descritivo, pautado em uma pesquisa de abordagem qualitativa através do método hipotético dedutivo.

Segundo Ludke e Andre (2013), nas pesquisas qualitativas o pesquisador se utiliza do ambiente natural como fonte de dados, nesse contexto, é necessário que tenham um contato direto e de longo prazo com o ambiente e a situação sob investigação. Geralmente este tipo de estudo ocorre por meio de intenso trabalho de campo, que se desenvolve a partir de um variado tamanho de amostras, importantes para gerar novas informações, aprofundadas e descritivas. Em suma, as pesquisas qualitativas são caracterizadas pelo cenário natural, por dados descritivos, em que o processo é predominante ao produto e as opiniões e processos são de cunho indutivo.

Com isso, buscou-se coletar informações em resposta à seguinte problemática da pesquisa: "O que a literatura mostra 
sobre os resultados do microagulhamento em cicatrizes de queimaduras?"

Para elaboração deste estudo foram reunidos artigos de caráter significativo e importante no campo científico sobre o tema proposto. A coleta de artigos foi realizada por meio de cinco itens, que são: definição de tema, seleção de base de dados, filtragem dos artigos, avaliação dos estudos e a construção dos resultados e discussões. A pesquisa foi realizada nas seguintes bases de dados eletrônicas: Google Acadêmico, Scientific Electronic Library Online (SciELO), Physiotherapy Evidence Database (PEDro), PubMed, utilizando os seguintes descritores: queimaduras, cicatriz e microagulhamento.

Para as análises, foi realizada a leitura criteriosa dos artigos selecionados. A partir da busca, encontrou-se um total de 4 artigos que corresponderam a temática deste estudo, os quais mostraram a eficácia do microagulhamento no tratamento de cicatrizes causadas pela queimadura.

Os critérios de inclusão foram artigos que relatavam acerca do uso da técnica de microagulhamento em cicatrizes de queimaduras, que utilizavam a técnica em cicatrizes de $2^{\circ}$ e $3^{\circ}$ de queimadura, publicados nos anos de 2011 a 2021 , tanto na língua inglesa, quanto portuguesa.

Como critérios de exclusão, artigos provenientes de revisão de literatura, revisão bibliográfica, teses acadêmicas, trabalhos duplicados com o período de tempo anterior à 2011, com títulos repetidos, ou que não abordavam o tema.

\section{Resultados e Discussão}

No Quadro 1 mostra-se 2 estudos realizados em indivíduos de ambos os sexos, 1 estudo realizado somente em uma paciente mulher e 1 estudo realizados somente em 1 paciente homem, submetidos ao microagulhamento. 
Quadro 1 - Resultado dos estudos publicados nos anos de 2011 a 2021.

\begin{tabular}{|c|c|c|c|c|c|}
\hline Titulo & Autor & Amostra & $\begin{array}{c}\text { Graus da } \\
\text { Queimadura }\end{array}$ & Objetivos & Resultados \\
\hline $\begin{array}{c}\text { A Atuação da } \\
\text { Fisioterapia no } \\
\text { Tratamento de } \\
\text { Sequela de } \\
\text { Queimaduras por } \\
\text { Meio da Indução } \\
\text { Percutânea de } \\
\text { Colágeno: Estudo } \\
\text { de Caso }\end{array}$ & $\begin{array}{l}\text { (Arantes, } \\
\text { et al., } \\
2020 \text { ) }\end{array}$ & $\begin{array}{l}1 \text { paciente de } 54 \\
\text { anos, queimadura por } \\
\text { óleo quente, há dois } \\
\text { anos, região do } \\
\text { membro superior } \\
\text { esquerdo. }\end{array}$ & $\begin{array}{l}\text { Queimadura } \\
\text { de } 2^{\circ} \text { grau }\end{array}$ & $\begin{array}{l}\text { Teve como objetivo a } \\
\text { aplicação da técnica } \\
\text { de Indução } \\
\text { Percutânea de } \\
\text { Colágeno (IPC) em } \\
\text { uma cicatriz causada } \\
\text { por queimadura de } \\
\text { segundo grau } \\
\text { profundo na região } \\
\text { anterior do braço } \\
\text { esquerdo. }\end{array}$ & $\begin{array}{l}\text { Os resultados obtidos pela escala de } \\
\text { Vancouver revelaram melhora, } \\
\text { sendo que o aspecto avaliado que } \\
\text { mais apresentou melhora foi a } \\
\text { flexibilidade da pele, seguido da } \\
\text { vascularização e altura da cicatriz. }\end{array}$ \\
\hline $\begin{array}{l}\text { Agulhamento } \\
\text { médico: efeito } \\
\text { sobre o eritema da } \\
\text { pele de cicatrizes } \\
\text { de queimaduras } \\
\text { hipertróficas. }\end{array}$ & $\begin{array}{l}\text { (Busch, } \\
\text { et al., } \\
2018 \text { ) }\end{array}$ & $\begin{array}{l}20 \text { pacientes com } \\
\text { idade média de } 34 \text { à } \\
64 \text { anos. }\end{array}$ & $\begin{array}{l}\text { Queimaduras } \\
\text { de } 2^{\circ} \text { e } 3^{\circ} \\
\text { grau. }\end{array}$ & $\begin{array}{l}\text { O objetivo do estudo } \\
\text { foi examinar a } \\
\text { influência do } \\
\text { agulhamento médico } \\
\text { no eritema } \\
\text { patológico e } \\
\text { persistente de } \\
\text { cicatrizes de } \\
\text { queimaduras } \\
\text { hipertróficas. } \\
\end{array}$ & $\begin{array}{l}\text { As cicatrizes examinadas mostraram } \\
\text { uma redução significativa do } \\
\text { eritema e ficaram menos } \\
\text { avermelhadas após o tratamento. } \\
\text { Com base nos resultados de } \\
\text { medições objetivas, a agulhagem } \\
\text { médica atingiu uma normalização } \\
\text { da cor da pele e um ajuste à pele } \\
\text { saudável após tratamentos } \\
\text { repetitivos. }\end{array}$ \\
\hline $\begin{array}{l}\text { Efeitos da Terapia } \\
\text { por Indução de } \\
\text { Colágeno na } \\
\text { qualidade de } \\
\text { cicatrizes de } \\
\text { queimaduras - } \\
\text { Relato de caso. }\end{array}$ & $\begin{array}{l}\text { (Tizatto, } \\
\text { et al., } \\
2015)\end{array}$ & $\begin{array}{l}\text { Paciente de } 27 \text { anos, } \\
\text { sexo masculino, } \\
\text { mecânico, não } \\
\text { tabagista, sem } \\
\text { presença de afecções } \\
\text { associadas, com } \\
\text { histórico de } \\
\text { cicatrização normal, } \\
\text { sem presença de } \\
\text { afecções dérmicas ou } \\
\text { propensão à } \\
\text { queloides. }\end{array}$ & $\begin{array}{l}\text { Queimaduras } \\
\text { de } 2^{\circ} \text { grau. }\end{array}$ & $\begin{array}{l}\text { O presente relato de } \\
\text { caso teve por } \\
\text { objetivo mostrar os } \\
\text { efeitos da Terapia } \\
\text { por Indução de } \\
\text { Colágeno na } \\
\text { qualidade de } \\
\text { cicatrizes de } \\
\text { queimaduras. }\end{array}$ & $\begin{array}{l}\text { Em todos os instrumentos } \\
\text { utilizados, houve melhora dos } \\
\text { aspectos analisados da cicatriz, } \\
\text { incluindo a aparência, por meio das } \\
\text { fotografias, que mostraram } \\
\text { resultados positivos. Além disso, o } \\
\text { paciente referiu melhora da } \\
\text { satisfação pessoal em relação aos } \\
\text { resultados obtidos. }\end{array}$ \\
\hline $\begin{array}{l}\text { Terapia percutânea } \\
\text { de indução de } \\
\text { colágeno: um } \\
\text { tratamento } \\
\text { alternativo para } \\
\text { cicatrizes de } \\
\text { queimaduras }\end{array}$ & $\begin{array}{l}\text { (Aust, et } \\
\text { al., 2010) }\end{array}$ & $\begin{array}{l}16 \text { pacientes } \\
\text { consecutivos - idade } \\
\text { média: } 37 \text { anos, } \\
\text { índice de massa } \\
\text { corporal (IMC) } \\
\text { médio: } 25,7 .\end{array}$ & $\begin{array}{l}\text { Queimadura } \\
\text { de } 2^{\circ} \text { grau }\end{array}$ & $\begin{array}{l}\text { Este estudo teve } \\
\text { como objetivo } \\
\text { avaliar a indução } \\
\text { percutânea de } \\
\text { colágeno (ICP) em } \\
\text { cicatrizes pós- } \\
\text { queimadura. }\end{array}$ & $\begin{array}{l}\text { Em média, os pacientes avaliaram } \\
\text { sua melhora como uma média de } \\
80 \% \text { melhor }( \pm 15,5) \text { do que antes } \\
\text { do tratamento. O exame histológico } \\
\text { revelou aumento considerável na } \\
\text { deposição de colágeno e elastina } 12 \\
\text { meses após a cirurgia. A epiderme } \\
\text { demonstrou espessamento de } 45 \% \\
\text { do estrato espinhoso e cristas rete } \\
\text { normais, bem como a normalização } \\
\text { da matriz de colágeno/elastina na } \\
\text { derme reticular em } 1 \text { ano de pós- } \\
\text { operatório. }\end{array}$ \\
\hline
\end{tabular}

Fonte: Autoria própria (2021).

Mediante a revisão de literatura, foi possível observar a eficácia do microagulhamento em cicatrizes de queimaduras, proporcionando uma melhora visual no aspecto estético e contribuindo na autoestima dos pacientes, como relatado no quadro 1.

Para se obter resultados significativos, Arantes, et al., (2020) realizou seu estudo com uma (1) paciente voluntária de 54 anos, a qual sofreu uma queimadura de $2^{\circ}$. A paciente foi submetida a escala de Vancouver, onde a pontuação varia de 0 a 13. Nesta escala quanto menor a somatória da pontuação final, melhor apresenta-se o estado da cicatriz. O estudo também realizou fotodocumentação em diferentes momentos do tratamento.

Através dos resultados obtidos pela escala de Vancouver, o estudo de Arantes, et al., (2020) revelou melhoras significativas da aparência da cicatriz com o uso da indução percutânea de colágeno (IPC) por meio da técnica de microagulhamento, sendo que o aspecto avaliado que mais apresentou melhora foi a flexibilidade da pele, seguido da 
vascularização e altura da cicatriz. Não foi possível observar melhora na pigmentação da cicatriz através da escala de Vancouver, porém, o score total passou de 7 para 4 após a última sessão, diminuindo em 3 pontos no total (cerca de $43 \%$ ).

No estudo de Busch, et al., (2018), o microagulhamento pode sim ser uma boa opção para uma abordagem terapêutica adequada no tratamento de cicatrizes de queimaduras hipertróficas, pois, segundo relata o autor, o procedimento influencia na vascularização, estimulando a angiogênese na cascata de cura da ferida pós-necessitada. Como o método foi baseado na indução percutânea de colágeno, a síntese do colágeno melhorou a espessura vital da epiderme, que está diretamente associada a menos transparência.

As cicatrizes examinadas na pesquisa de Busch, et al., (2018) apresentaram redução significativa do eritema e mostraram-se menos avermelhadas após o tratamento. Com base nos resultados das medidas objetivas, a necessidade médica consegue uma normalização da cor da pele e um ajuste na pele saudável após tratamentos repetitivos.

Já no estudo de Tizatto, et al., (2015), os resultados referentes à evolução da cicatriz, após três sessões do tratamento, de acordo com a Escala de Vancouver, apresentaram escore inicial 9 e escore final 4, demonstrando melhora progressiva na elasticidade e altura da cicatriz. A escala não foi sensível para graduar a melhora da pigmentação e da vascularização, entretanto, de acordo com os resultados obtidos pela Escala de avaliação da cicatriz, foi possível observar uma melhora considerável do aspecto da cicatriz em todas as variáveis também de forma progressiva, apresentando escore -1 na avaliação inicial e escore 8 na avaliação final. Estima-se que houve um aumento considerável em relação à dor no decorrer das intervenções, caracterizando, desta forma, uma resposta positiva na sensibilidade cutânea.

As efetividades do estudo podem ser confirmadas através de respostas de questionário de satisfação, onde o participante relatou a melhora em todos os aspectos abordados, mostrou-se bastante satisfeito com os resultados obtidos, destacando estar mais feliz com sua autoimagem (Tizatto, et al., 2015).

Seguindo o mesmo direcionamento dos demais estudos que apresentaram resultados promissores acerca da problemática levantada na presente pesquisa, Aust, et al., (2010) fez a seleção de 16 pacientes de ambos os sexos, com idade de 15 a 35 anos, que foram acompanhados durante 12 meses. Quatro semanas antes do procedimento, os pacientes foram preparados com cremes cosméticos tópicos de vitamina A e C para maximizar a estimulação do colágeno e para a avaliação foi utilizada a escala de VSS que leva em consideração 4 parâmetros: vascularidade, altura (espessura), flexibilidade e pigmentação, onde a pontuação vai de 0 (pele normal) a 4 (mais grave).

A partir disso, após o procedimento na escala VSS, a pontuação pré-operatória média foi de 4,5 (variação, 2-5), que melhorou significativamente para 8,5 (intervalo, 7-10) no pós-operatório, a pontuação VSS pré-operatória média foi 7,5 Æ 11,5 (intervalo, 4-11), que melhorou para 4,8 Æ 15,5 (intervalo, 1-6), 1 ano após a terapia ICP. As pontuações da escala melhoraram em média de $27 \pm$ 13,5 (variação, 14-38) no pré-operatório para 19 Æ 11,5 (variação, 14-25), 1 ano após a ICP terapia. No pósoperatório, nenhum paciente experimentou qualquer fotossensibilidade ou desenvolveu qualquer pós-inflamatório hiperpigmentação ou hipopigmentação (Aust, et al., 2010).

Dando prosseguimento ao mesmo estudo, referente ao exame histológico no qual foi utilizada a coloração de Van Gieson, mostrou uma normalização considerável de matriz de colágeno/elastina da derme reticular e um aumento da deposição de colágeno aos 12 meses, após o procedimento. O colágeno também mostrou ter sido depositado de forma normal padrão de rede, ao invés de feixes paralelos, como visto na cicatriz de tecido. A coloração com hematoxilina e eosina demonstrou um normalestrato córneo, epiderme espessada (45\% de espessamento dostratum granulosum) e cristas rete normais 1 ano pósoperatório ativamente (Aust, et al., 2010).

A partir dos dados obtidos na pesquisa de Aust, et al., (2010), o microagulhamento, além de ser um método simples, é também eficaz, pois suas hipóteses primárias foram comprovadas, uma vez que a avaliação e exames histológicos revelam melhora significativa da cicatriz, sendo assim a IPC visa estimular a produção de colágeno, usando a cascata química que ocorre 
após qualquer tipo de trauma.

Como observado nos estudos apresentados no decorrer desta pesquisa, os autores concordam entre si no que diz respeito ao uso e eficácia da técnica aplicada para o tratamento de queimaduras, comprovando sua aplicabilidade. Verificou-se que o IPC em todos os estudos apresentou resultados positivos. Portanto, a partir da revisão de literatura, o tratamento revela um baixo custo, fácil aplicação e rápido tratamento, comparado à outras técnicas invasivas existentes no mercado para a fisioterapia dermatofuncional.

\section{Conclusão}

O microagulhamento ou a indução percutânea de colágeno (IPC) tem chamado muita a atenção das pessoas que acreditam em tratamentos clínicos estéticos no trato com a beleza da pele, sendo possível a redução de manchas, cicatrizes, rugas, estrias, entre outros benefícios.

Conclui-se então, que o microagulhamento apesar de ser uma técnica nova no mercado, vem ganhando o seu espaço, provando ser promissora e eficaz no tratamento das cicatrizes de queimaduras, promovendo a melhora do aspecto estético, assim como também da autoestima dos pacientes.

Apesar de poucos autores tratarem sobre esse assunto, o que resulta em uma grande escassez de dados científicos, estudos já comprovaram os efeitos benéficos dessa técnica, a qual pode ser realizada em todos os tipos de pele, em que o custo é menor em relação a outras técnicas, sendo possível desenvolver vários programas e planos de tratamento em diferentes áreas do corpo, incluindo áreas de difícil acesso.

Infere-se que a aplicação da técnica tem resultados em poucas sessões, e pode ser utilizada até mesmo no verão, o que acaba influenciando na autoestima, na melhora física e mental, proporcionando uma melhor qualidade de vida a esses pacientes.

Sugere-se então, a realização de novos estudos, como por exemplo, os ensaios clínicos randomizados duplo cegos, que são considerados de melhor qualidade, segundo a medicina baseada em evidências. Assim, torna-se imprescindível a realização de estudos com grupos de voluntários, estudos de caso, pesquisa de campo, estudos qualitativos e pesquisas que sejam realizados nesse padrão.

Além disso, são necessários novos estudos que aprofundem a temática discutida no escopo desta pesquisa, para que venham agregar ainda mais com a comunidade acadêmico-científica e, em particular neste estudo, na melhora de tratamentos mediados pela fisioterapia dermatofuncional envolvendo pacientes com queimaduras.

\section{Referências}

Arantes, et al., (2020). A Atuação da Fisioterapia no Tratamento de Sequela de Queimaduras por Meio da Indução Percutânea de Colágeno: Estudo de Caso. Revista Pluri, 14(1), 49-53. doi:10.26843/rpv132020p149-156.

Aust, et al., (2010). Terapia percutânea de indução de colágeno: um tratamento alternativo para cicatrizes de queimaduras. Burns, 36 (1) 836-843.

Baryza, M. J. (1995). The Vancouver Scar Scale: an administration tool and its interrater reliability. J Burn Care Rehabil, 16(5), 535-538.

Busch, K, et al., (2018). Agulhamento médico: efeito sobre o eritema da pele de cicatrizes de queimadura hipertrófica. Cureus10(9), e3260. doi:10.7759/cureus.3260.

Dangello, J. G., \& Fattini, C. A. (1998). Anatomia humana sistêmica e segmentar para o estudante de medicina. (2a ed.), Atheneu.

Doddaballapur, S. (2009). Microneedling with Dermaroller. Journal of Cutaneous and Aesthetic Surgery, 2(2), 110-111. http://www.ncbi.nlm.nih.gov/pmc/articles/PMC2918341/?report=printable.

Ferreira, L. M. (2007). Cirurgia Plástica. Manole.

Guirro, E. \& Guirro, R. (2007). Fisioterapia DermatoFuncional: Fundamentos, Recursos e Patologias. Manole.

Guirro, E. C. O., \& Guirro, R. R. J. (2004). Noções de Citologia e Histologia. Fisioterapia Dermato - Funcional: Fundamentos, Recursos, Patologia. (3a ed.), revisada e ampliada-Barueri. Manole. 
Research, Society and Development, v. 10, n. 14, e167101421974, 2021

(CC BY 4.0) | ISSN 2525-3409 | DOI: http://dx.doi.org/10.33448/rsd-v10i14.21974

Harris, M. I. N. (2016). Pele: do nascimento à maturidade. Editora Senac.

Junqueira, L. C., \& Carneiro, J. (2008). Histologia Básica. (11a ed.), Guanabara Koogan.

Kalil, C. L. P. V., et al., (2015). Tratamento das cicatrizes de acne com a técnica de microagulhamento e drug delivery. Surgical \& Cosmetic Dermatology, 7(2), 144-148. 10.5935/scd1984-8773.201572641.

Lamberti, D. P, et at., (2014). Recursos fisioterapêuticos em paciente queimado: relato de caso de um sobrevivente do incêndio na boate kiss. Rev uningá.;18(2): 38-41. http://revista.uninga.br/index.php/uningareviews/article/view/1504.

Liebl, H., \& Kloth, L. C. (2012). Skin Cell Proliferation Stimulated by Microneedles. Journal of the American College of Clinical Wound Specialists, Milwaukee, 4 (1), 2-6. http://www.jaccws.org/article/S22135103(12)00072-3/pdf .

Lima, E. V. A, et al., (2013). Microagulhamento: estudo experimental. Surgical \& Cosmetic Dermatology, 5 (2), 110-114. http://www.redalyc.org/articulo.oa?id=265527948004.

Ludke, M. \& Andre, M. E. D. A. (2013). Pesquisas em educação: uma abordagem qualitativa. E.P.U.

Moren, S. A., (2009). SPAs e salões de beleza terapias: Terapias Passo a Passo. Cengage.

Negrão, M. M. C. (2015). Microagulhamento: bases fisiológicas e práticas. CR8 Editora.

Serra, M. C. V. F., et al., (2006). Fisiologia e fisiopatologia. In: Lima, J.R \& Maciel, E. Tratado de queimaduras. Ateneu, p. 37.

Souza, N. S. (2019). Os benefícios do microagulhamento no tratamento de disfunções estéticas. https://faculdadefacsete.edu.br/monografia/items/show/568.

Tizatto, et al., (2015). Efeitos da Terapia por Indução de Colágeno na qualidade de cicatrizes de queimaduras - Relato de caso. Pluri. Relações África-Brasil: cotidiano, sociedade e experiências religiosas, 3, 149- 156.

Vana, L. P. M. (2013). Sequelas de queimaduras: nova classificação. Rev. Bras. Queimaduras, 12 (3), 192-2. 